OPEN ACCESS

Edited by:

Andrew P. Lavender

Federation University

Australia, Australia

Reviewed by:

Ulrich Bickel,

Texas Tech University Health Sciences

Center, United States

Berislav Zlokovic,

University of Southern California,

United States

${ }^{*}$ Correspondence:

Craig F. Ferris

c.ferris@northeastern.edu

†These authors have contributed equally to this work

Specialty section:

This article was submitted to Neurotrauma,

a section of the journal

Frontiers in Neurology

Received: 23 June 2021

Accepted: 24 August 2021

Published: 30 September 2021

Citation:

Leaston J, Qiao J, Harding IC Kulkarni P, Gharagouzloo C, Ebong E and Ferris CF (2021) Quantitative

Imaging of Blood-Brain Barrier Permeability Following Repetitive Mild Head Impacts.

Front. Neurol. 12:729464. doi: 10.3389/fneur.2021.729464

\section{Quantitative Imaging of Blood-Brain Barrier Permeability Following Repetitive Mild Head Impacts}

\author{
Joshua Leaston ${ }^{1 \dagger}$, Ju Qiao ${ }^{2 \dagger}$, Ian C. Harding ${ }^{3}$, Praveen Kulkarni ${ }^{1}$, Codi Gharagouzloo ${ }^{1,2}$, \\ Eno Ebong ${ }^{4}$ and Craig F. Ferris ${ }^{2,5 *}$ \\ ${ }^{1}$ Imaginostics, Inc., Cambridge, MA, United States, ${ }^{2}$ Center for Translational Neuroimaging, Northeastern University, Boston, \\ MA, United States, ${ }^{3}$ Department of Bioengineering, Northeastern University, Boston, MA, United States, ${ }^{4}$ Department of \\ Chemical Engineering, Northeastern University, Boston, MA, United States, ${ }^{5}$ Departments of Psychology and \\ Pharmaceutical Sciences, Northeastern University, Boston, MA, United States
}

This was an exploratory study designed to evaluate the feasibility of a recently established imaging modality, quantitative ultrashort time-to-echo contrast enhanced (QUTE-CE), to follow the early pathology and vulnerability of the blood brain barrier in response to single and repetitive mild head impacts. A closed-head, momentum exchange model was used to produce three consecutive mild head impacts aimed at the forebrain separated by $24 \mathrm{~h}$ each. Animals were measured at baseline and within $1 \mathrm{~h}$ of impact. Anatomical images were collected to assess the extent of structural damage. QUTE-CE biomarkers for BBB permeability were calculated on 420,000 voxels in the brain and were registered to a bilateral 3D brain atlas providing site-specific information on 118 anatomical regions. Blood brain barrier permeability was confirmed by extravasation of labeled dextran. All head impacts occurred in the absence of any structural brain damage. A single mild head impact had measurable effects on blood brain barrier permeability and was more significant after the second and third impacts. Affected regions included the prefrontal ctx, basal ganglia, hippocampus, amygdala, and brainstem. Our findings support the concerns raised by the healthcare community regarding mild head injuries in participants in organized contact sports and military personnel in basic training and combat.

\footnotetext{
Keywords: concussion, ferumoxytol, cerebral small vessel disease, momentum exchange, quantitative ultrashort time-to-echo, contrast enhanced MRI
}

\section{INTRODUCTION}

There is a growing concern and expanding literature on the behavioral and neurobiological consequences of repetitive mild head impacts or concussions incurred while playing organized sports or in military combat. Mild head impacts are estimated to account for $75 \%$ of all traumatic brain injuries (1). Concussion following a single incident is difficult to detect and any associated cognitive and behavioral problems can resolve within hours of insults $(2,3)$. However, a more pernicious, long-lasting condition arises when the brain is exposed to repeated mild head impacts $(4,5)$. Repetitive head impacts induce cognitive, motor and behavioral deficits, which are more severe and protracted, and can last for months and even years $(6,7)$ with an increased risk of dementia, and chronic traumatic encephalopathy $(\mathrm{CTE})(8,9)$. 
Failure in the blood brain barrier (BBB) lies at the foundation of cerebrovascular dysfunction as first described by Wardlaw (10). BBB failure is characterized by hyperpermeability of endothelial walls, damage to basement membranes, and enlargement of surrounding perivascular space allowing protein, macrophage, and lymphocyte invasion and $\beta$-amyloid $(A \beta)$ deposition $(11,12)$. Disruption in the BBB commonly occurs with moderate to severe traumatic brain injury (TBI) (13-17) and the insult may persist for years contributing to the neuropathology of neurodegenerative diseases (18). Animal models of repetitive mild head impact report no effect on BBB permeability $(19,20)$, or a modest increase that persists up to 3 days post insult $(21,22)$. Additionally, it has been documented in literarture that an early response to TBI may be a decrease in cerebral blood flow $(23,24)$, and it is speculated that this may play an important role in inhibiting the recovery process of repetitive mild TBI (rmTBI).

There are multiple imaging protocols for detecting the gross lesions that result from the neuropathological consequences of cerebral vascular injury, such as T2 Fluid Attenuated Inversion Recovery (FLAIR), Susceptibility Weighted Imaging (SWI), and Diffusion-Weighted Imaging (DWI). However, these methods cannot quantitatively assess BBB integrity (25). The most common way for assessing BBB leakage is dynamic contrast enhanced (DCE) MRI with gadolinium-based contrast agents (GBCAs) (26), particularly with respect to BBB permeability following head injury (27). DCE-MRI is limited in error in the arterial input function (AIF) $(28,29)$ and significant variances are reported for rates of leakage (30). More recently, higher accuracy measurements have been achieved in rodents $(31,32)$ and humans $(33,34)$, however DCE-MRI remains limited to 2-dimensional imaging with thick slabs $(1 \mathrm{~mm}$ in mice, $5 \mathrm{~mm}$ in humans) - in addition to requiring toxic GBCAs which have recently obtained an FDA black-box warning for brain retention in 2017. To address the need for safe, quantitative, whole-brain non-invasive precision medicine diagnostics for mild brain injury, we explored the use of a recently established alternative technique quantitative ultrashort time-to-echo contrast enhanced (QUTE-CE) MRI (35-39). This method has recently been utilized to map BBB leakage due to Type-2 Diabetes (38). Here, for the first time, we report the use of this technique to measure BBB leakage at the individual animal level, and for head injury, in a model of repeated mild impacts.

\section{MATERIALS AND METHODS}

\section{Animals}

Subjects were all adult male Sprague Dawley rats $(n=5), \sim 100$ days of age and purchased from Charles River Laboratories (Wilmington, MA, USA). Animals were housed in Plexiglas cages and maintained in ambient temperature $\left(22-24^{\circ} \mathrm{C}\right)$ on a $12: 12$ light-dark cycle (lights on at 07:00 a.m.). Food and water were provided ad libitum. All methods and procedures described were approved by the Northeastern University Institutional Animal Care and Use Committee (IACUC). The Northeastern facility is AAALAC accredited with OLAW Assurance and is registered with the USDA. All housing, care, and use followed the Guide for the Care and Use of Laboratory Animals (8th Addition) and the Animal Welfare Act. The protocols used in this study adhere to the ARRIVE guidelines for reporting in vivo experiments in animal research (40).

\section{Momentum Exchange Model}

Concussion were generated with a pneumatic pressure drive, $50 \mathrm{~g}$ compactor described by Viano et al. (41) and refined by Mychasiuk et al. (42) to reliably produce the $7.4 \mathrm{~m} / \mathrm{s}$ impact velocities described for mild rat head injury. The kinetic energy at impact is 1.37 joules. We have used this model to publish on the long-term neuroradiological effects of repetitive mild head impacts in rats (43). The impact piston was directed to the top of the skull, midline, in the approximate area of Bregma (Supplementary Figure 2A) while rats were anesthetized under $1-2 \%$ isoflurane. Rats were awake and ambulatory within 5$7 \mathrm{~min}$ after anesthesia and concussion.

\section{Imaging}

Studies were done on a Bruker Biospec 7.0T/20-cm USR horizontal magnet (Bruker, Billerica, MA, USA) and a 20$\mathrm{G} / \mathrm{cm}$ magnetic field gradient insert (ID $=12 \mathrm{~cm}$ ) capable of a $120-\mu$ s rise time. Radio frequency signals were sent and received with a quadrature volume coil built into the rat restrainer (Ekam Imaging, Boston, MA, US). All rats imaged under $1-2 \%$ isoflurane while keeping a respiratory rate of $40-$ 50 breadths/min.

Animals were measured for baseline prior to being subjected to any head impact. Beginning $24 \mathrm{~h}$ later, each rat was subjected to three mild head impacts separated by $24 \mathrm{~h}$ intervals as previously described (43). All rats were imaged within $1 \mathrm{~h}$ of head impact. Following the final impact, rats were anesthetized with isoflurane exposure and transcardially perfused for postmortem histology.

At the beginning of each imaging session, a high-resolution anatomical data set was collected using the RARE pulse sequence with following parameters, 35 slice of $0.7 \mathrm{~mm}$ thickness; field of view [FOV] $3 \mathrm{~cm} ; 256 \times 256$; repetition time [TR] 3,900 ms; effective echo time [TE] $48 \mathrm{~ms}$; number of excitations (NEX) 3; $6 \mathrm{~min} 14 \mathrm{~s}$ acquisition time. Rats were imaged prior to and following an i.v. bolus of $6 \mathrm{mg} / \mathrm{ml} \mathrm{Fe}$ of Ferumoxytol. The injected volume was tailored for each rat (assuming $7 \%$ blood by body weight) to produce a starting blood concentration of $200 \mu \mathrm{g} / \mathrm{ml} \mathrm{Fe}$ (twice the clinical dose approved for use in humans). 3D-UTE image acquisition parameters were TE $=13$ $\mu \mathrm{s}, \mathrm{TR}=4 \mathrm{~ms}$, and flip angle $(\mathrm{FA})=20^{\circ}$ with a RF hard pulse bandwidth of $200 \mathrm{kHz}$. Therefore, the pulse duration was short $(6.4 \mu \mathrm{s})$ compared to the T2 of the approximate ferumoxytol concentration $(4.4 \mathrm{~ms}$ for $3.58 \mathrm{mM}$, i.e., $200 \mu \mathrm{g} / \mathrm{ml})$ to minimize signal blur and reduce the probability for a curved trajectory of the magnetization vector Mz. A $3 \times 3 \times 3 \mathrm{~cm}^{3}$ field-of-view was used with a matrix mesh size of $180 \times 180 \times 180$ to produce $167 \mu \mathrm{m}$ isotropic resolution. Example images from scan sessions can be seen in Supplementary Figure 1.

\section{Image Processing}

Images were motion-corrected, aligned spatially, and resliced with nearest neighbor algorithms to preserve original intensity 
values using MATLAB SPM12 toolbox develop at UCL (http:// www.fil.ion.ucl.ac.uk/spm/). The T2-weighted RARE anatomical data for each rat taken at each imaging session was fit to a 3D MRI Rat Brain Atlas (C) (Ekam Solutions LLC, Boston, MA, US) with 118 segmented annotated brain areas using software developed at Northeastern University's Center for Translational Neuroimaging (CTNI). The fitted atlas was used for region-ofinterest (ROI) selection to determine site-specific abnormality. Image intensity was corrected for $\mathrm{B}^{-}$coil sensitivity along the $\mathrm{Z}$-axis using a homogenous copper sulfate 10 - $\mathrm{ml}$ tube phantom. Anatomical description of atlas regions by cluster can be found in Supplementary Table 1.

\section{BBB Permeability Biomarker}

In this experimental design, quantification of the $\mathrm{BBB}$ permeability was achieved by analyzing the slope of the $\mathrm{CBV}$ vs. time curve over 7 consecutive post-contrast scans (see Supplementary Figure 2B). Fluctuations in CBV in each region of interest was attributed to modulations in $\mathrm{BBB}$ permeability, and were calculated by the percentage change in apparent $\mathrm{CBV}$ per second.

\section{Statistical Analysis}

Group analyses were conducted with one-tailed repeated measure Analysis of Variance (rmANOVA), followed by several planned pairwise comparisons to assess the effect of repetitive mild head impacts on each brain region. Using this model, all subsequent scanning sessions were statistically compared to baseline to isolate the effect of head impacts on the BBB. To control for the effect of both type-I error and type-II error, multiple comparisons correction was applied through the Benjamin-Hochberg procedure with a false discovery rate $(p$ $<0.05 \&$ FDR $=0.1$ ) for each family of hypotheses. Random missing data was accounted for with mean imputation.

To determine if increases in BBB permeability could be detected at the individual animal level, the slope of each animal's CBV was analyzed over 7 scans with one-tailed linear regression $(p<0.1)$. This analysis was accompanied by Bonferroni correction predicated on the number of statistical tests in each data sample.

Blood brain barrier permeability (BBB) quantification were analyzed by the fitment of an atlas with 118 regions of interest. All significant individual animal results were assessed daily, and group averages were calculated to compare the $\mathrm{BBB}$ of each subsequent day to the respective control values.

All analyses were performed in MATLAB, SPSS version 27, and GraphPad Prism.

\section{Histological Analysis}

Changes in BBB permeability following mild impacts was confirmed via injection of FITC-fluorescent dextran, as previously described (44). Briefly, $70 \mathrm{kDa}$ FITC-dextran was injected intravenously to rats immediately after imaging. One hour after injection, rats were perfused and their brains collected, post fixed in $4 \%$ PFA for $24 \mathrm{~h}$, cryopreserved in $30 \%$ sucrose for $48 \mathrm{~h}$, and subsequently sectioned in 40-micron increments. Sections were stained with Lycopersicon esculentum (Tomato) lectin to label the vasculature and imaged using a laser scanning confocal microscope. Increased FITC signal in the perivascular space was indicative of increased BBB permeability. Permeability was quantified by analyzing mean fluorescent intensity of FITC-dextran outside of the vasculature (44). Images were taken using a Zeiss LSM 880 laser scanning confocal microscope in Northeastern University's Institute for Chemical Imaging of Living Systems. Regions found with increased BBB permeability with QUTE-CE imaging (orbital and motor cortices) were compared to areas that demonstrated no significant permeability differences (substantia nigra).

\section{Data Availability}

Data for average CBV values for all animals, days, scans, and region can be accessed at https:/github.com/Gharagouzloo/ FrontiersinNeurology2021.

\section{RESULTS}

\section{Structural Results}

There was no evidence of structural brain damage after mild head impact at any of the time points for any of the rats (Figure 1). The approximate site of the impact (red arrow) can be identified by the high intensity signal reflecting the edema on the tissue above the skull (black encasement of brain denoted by the yellow arrow). While the edema is apparent in most of the images, it is not obvious in Rat 3 after the 1st mild head impact and only slightly so in Rat 2 and Rat 5 . Nonetheless, Rat 2 showed a diffuse pattern of increased brain permeability after the 1st impact while Rats 3 and 5 showed very little (see Figure 2).

\section{Subject Site-Specific Changes in Blood Brain Permeability}

Figure 2 depicts heat maps showing the location of increases in $\mathrm{BBB}$ permeability for all animals (columns) prior to and following head impacts (rows). Data was acquired $1 \mathrm{~h}$ after each mild head impact. Data is missing (N/D) from Rat 4 from the 2nd impact because of technical issues. The coronal sections enable the visualization of the lateralization of $\mathrm{BBB}$ permeability changes (e.g., Rat 1: prefrontal, anterior, and retrosplenial cortices; Rat 3: striatum). The variability within and between subjects over the three impacts is also displayed. For example, Rats 1, 2, and 4, present with increased BBB permeability after the first impact, while Rats 3 and 5 do not. Indeed, Rat 5 appears resilient to the repetitive impacts, only showing a modest increase in $\mathrm{BBB}$ permeability in the somatosensory cortex and prefrontal cortex after the 3rd impact (see Supplementary Table 1, Supplementary Figure 3). The box and whisker plot shows the average permeability across rows for each condition as a percentage of the whole brain. Baseline permeability without impact was less than $1 \%$ of the total brain permeability (Figure 3). The greatest significant increase in permeability occurred after the 2 nd and 3rd impacts as compared to no impact. 


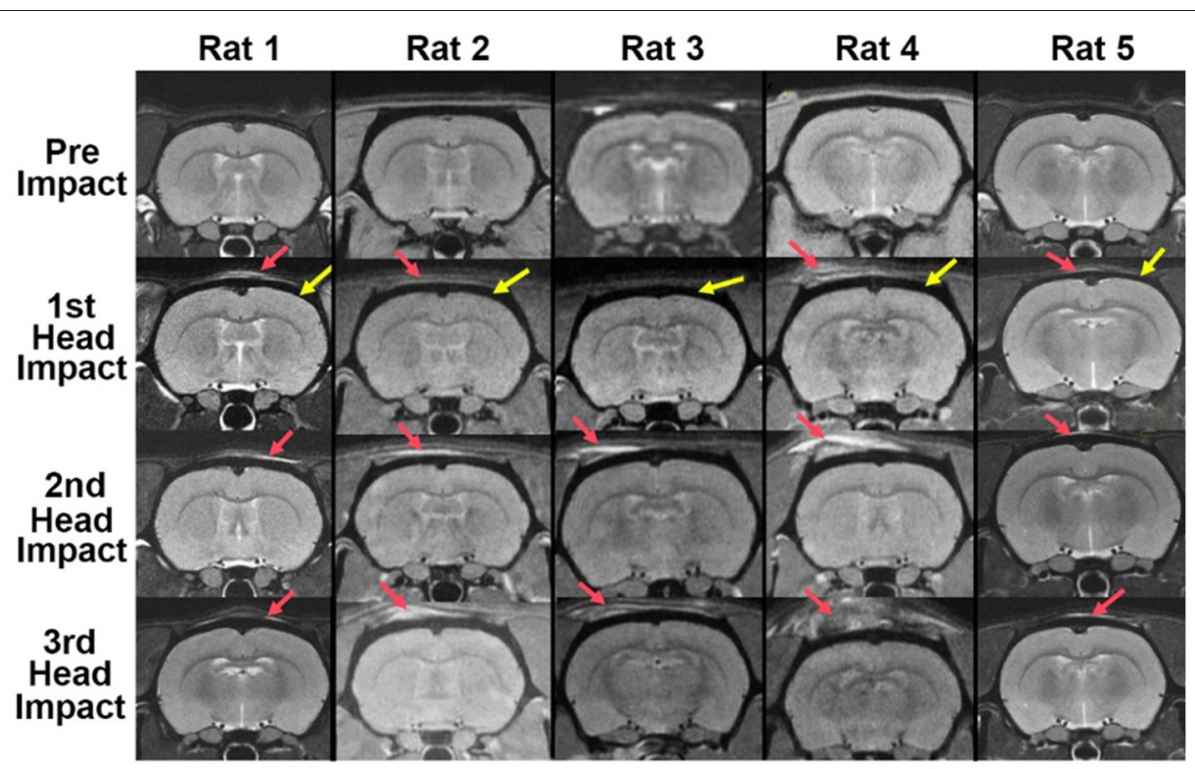

FIGURE 1 | Brain neuroanatomy following repetitive mild head impacts. Mild head impact without evidence of brain damage. Shown are axial sections of T2-weighted anatomical images taken within $1 \mathrm{~h}$ of mild head impact. The site of impact noted by the red arrow can be identified by the high intensity signal reflecting the edema on the tissue. The yellow arrows point to the black contour of the cranium. There is no visible evidence of structural brain damage.

\section{Subject-Specific Changes in Blood-Brain Barrier Permeability}

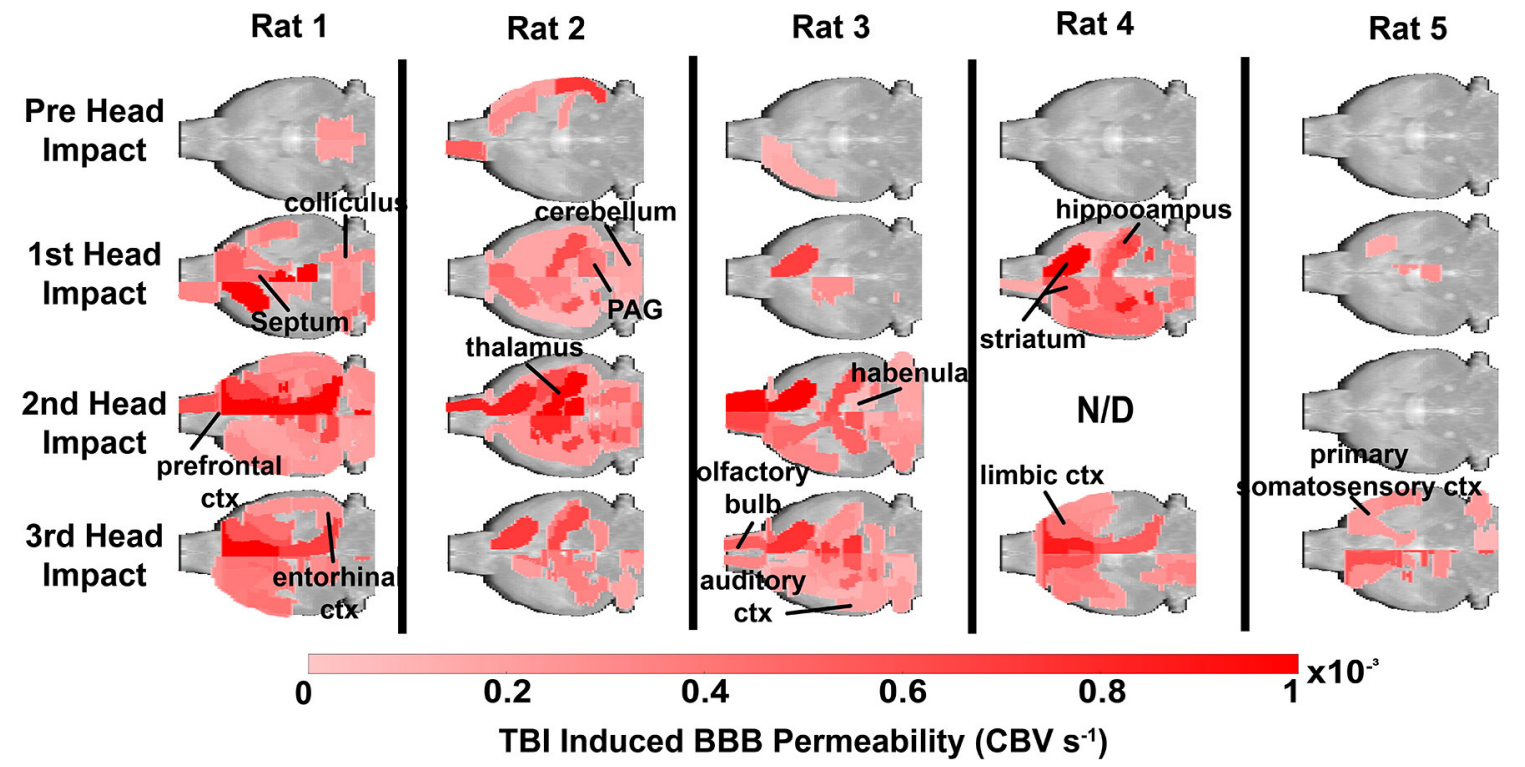

FIGURE 2 | Subject site-specific changes in blood brain barrier permeability. Each animal was scanned at baseline and following mild head impacts (1/day). Significance was determined by a linear regression model to assess the slope over the course of each imaging session, and then adjusted with a Bonferroni correction. Notably, increased BBB permeability was found near the site of impact. The color scale represents the slope and is only displayed if significant $(p<0.1$, FDR $=0.1)$.

\section{Group Site-Specific Changes in Blood Brain Barrier Permeability}

Heat maps depicting the site-specific location of the average increase in BBB permeability for all subjects are shown in Figure 4. The sagittal sections above show the progressive increase in permeability with repetitive mild head impacts starting in the forebrain with the prefrontal cortex and anterior olfactory tubercles and extending caudally to the retrosplenial ctx, colliculi, and pons. The coronal sections aligned dorsal (top) to ventral (bottom) below show the lateralization and exacerbated 


\section{Daily Whole-Brain BBB Permeability}

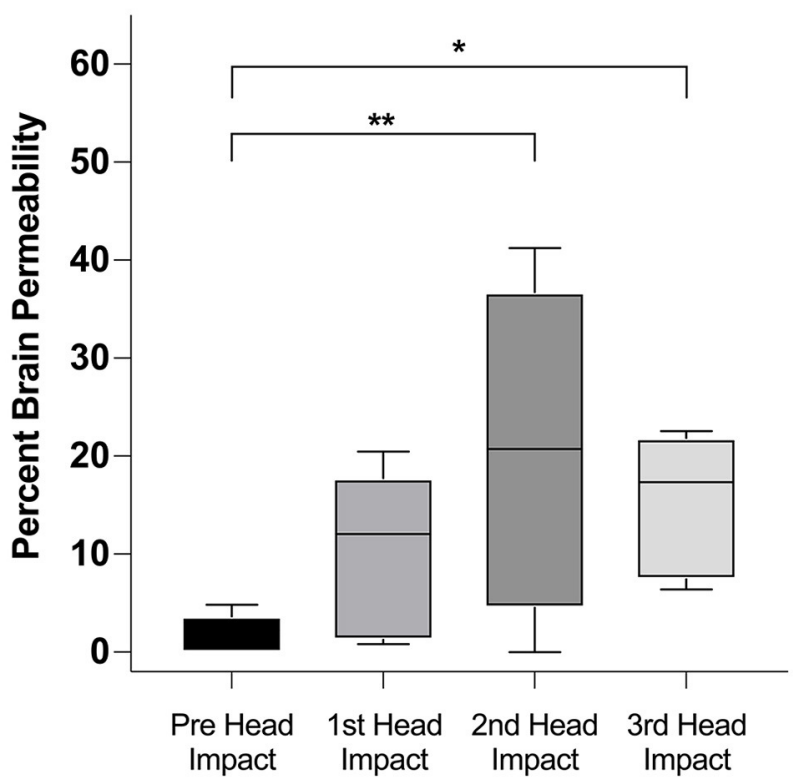

Day (each day = 1 hit)

FIGURE 3 | Whole-brain comparison of percent brain volume affected in subjects. After ascertaining if a region was affected in each animal (Figure 2) we used a one-tailed mixed effect analysis $(p<0.05 ; \mathrm{FDR}=0.1)$ and Benjamin Hochberg correction to determine if the affected brain volumes changed between days. Significance in this figure is represented by asterisks $\left({ }^{*} p<0.05,{ }^{* *} p<0.01\right)$.

permeability with repeated head impacts as exemplified by the 2nd mild head impact (e.g., row b. M2, M1, CG, S1UL, and S2). Table 1 shows all the areas that have a significant increase in $\mathrm{BBB}$ permeability for each of the mild head impacts and their lateralization to either the right or left side of the brain. All the head impacts include increased BBB permeability in the olfactory system including the olfactory tubercles $(\mathrm{Tu})$, tenia tecta $(\mathrm{TT})$, piriform ctx (Pir), endopiriform ctx (En), and anterior olfactory nucleus $(\mathrm{AON})$. The caudate putamen $(\mathrm{CPu})$ or striatum is also affected with each mild head impact. With the 3rd mild impact, the increased permeability appears in hindbrain areas that include the hippocampus (row c. DG -dentate gyrus, row b. DS-dorsal subiculum, row c. CA1d, e. CA1v), inferior colliculus (IC), principle trigeminal nucleus ( $\operatorname{Pr} 5)$, and vestibular nucleus $(\mathrm{Ve})$. Progressive BBB permeability is observed from Day 1 at $2 \%$, Day 2 at $7 \%$ and Day 3 at 19\% whole-brain BBB permeability (Supplementary Figure 4, Supplementary Table 2).

\section{Histology}

Brain regions with increased permeability as identified by QUTE-CE were validated via FITC-dextran injection (Figure 5). Fluorescent imaging and quantitative image analysis demonstrated that repetitive mild head impacts led to an increase in $\mathrm{BBB}$ permeability in both the second motor and orbital cortex (Figures 5A,B,D). This is highlighted by increased dextran accumulation in the brain parenchyma (white arrows) as well as within vascular cells, potentially suggesting increased retention by endothelial cells. In contrast, low levels of parenchymal and vascular dextran were observed in the substantia nigra (Figures 5C,D). These results agree with QUTE-CE results.

\section{DISCUSSION}

The present study followed the immediate and accumulative effects of one, two, and three mild head impacts on site-specific changes in $\mathrm{BBB}$ permeability over the entire brain in rats both at the individual and group levels. At the group level, only a modest effect was displayed after one hit, and the consequences of a second and third hit were more severe, affecting a greater area of the brain. These results are discussed with respect to the clinical implications of mild repetitive head impacts without structural brain damage and the use of a novel imaging modality, QUTE-CE MRI, to identify these putative changes in injuryinduced permeability.

There are numerous studies reporting moderate to severe traumatic brain injury (TBI) can have long-term deleterious effects on BBB integrity and function (18). There are numerous human and animal studies using various in-vivo and in-vitro methods showing that repetitive mild TBI separated by short intervals of time posse a significant risk to the brain and vulnerability to neurodegenerative disorders with aging (4548). Indeed, the cerebral vascular injury from TBI impacts the function of the neurovascular unit and may be a contributing factor in the pathophysiology of neurodegenerative diseases (49, 50). Indeed, moderate to severe TBI is a risk factor for Alzheimer's (51, 52), Parkinson's (53) and Amyotrophic Lateral Sclerosis (54). However, mild head injury is the most prevalent form of head injury and is estimated to affect over 42 million people worldwide every year due mostly to falls and motor accidents $(1,7)$. Repetitive mild head impacts in organized sports or on the battlefield have raised concerns about their long-term effects on neurodegeneration, e.g., CTE (9). The health community lists guidelines for diagnosing these mild head injuries that include self-reports of transient confusion, disorientation, impaired consciousness, or dysfunction in memory around the time of the injury and, importantly, no apparent structural damage as determined with imaging $(1,55,56)$. Unfortunately, many existing models for TBI in animals are far too severe and therefore do not mimic the more insidious effect of mild impact.

In our model, the skull was intact for all animals, and there was no radiological evidence of a contusion, or structural damage, but only edema near the site of impact. There are several studies on the neurobiological effects of single and repetitive head impacts in closed head models-these are not considered TBI because there is no structural brain damage. For example, Kane and coworkers assessed the effects of repetitive mild head impacts on over 300 mice using measures of behavior and postmortem histology (19). With a closed head momentum exchange model, they delivered a single impact over five consecutive days. The 


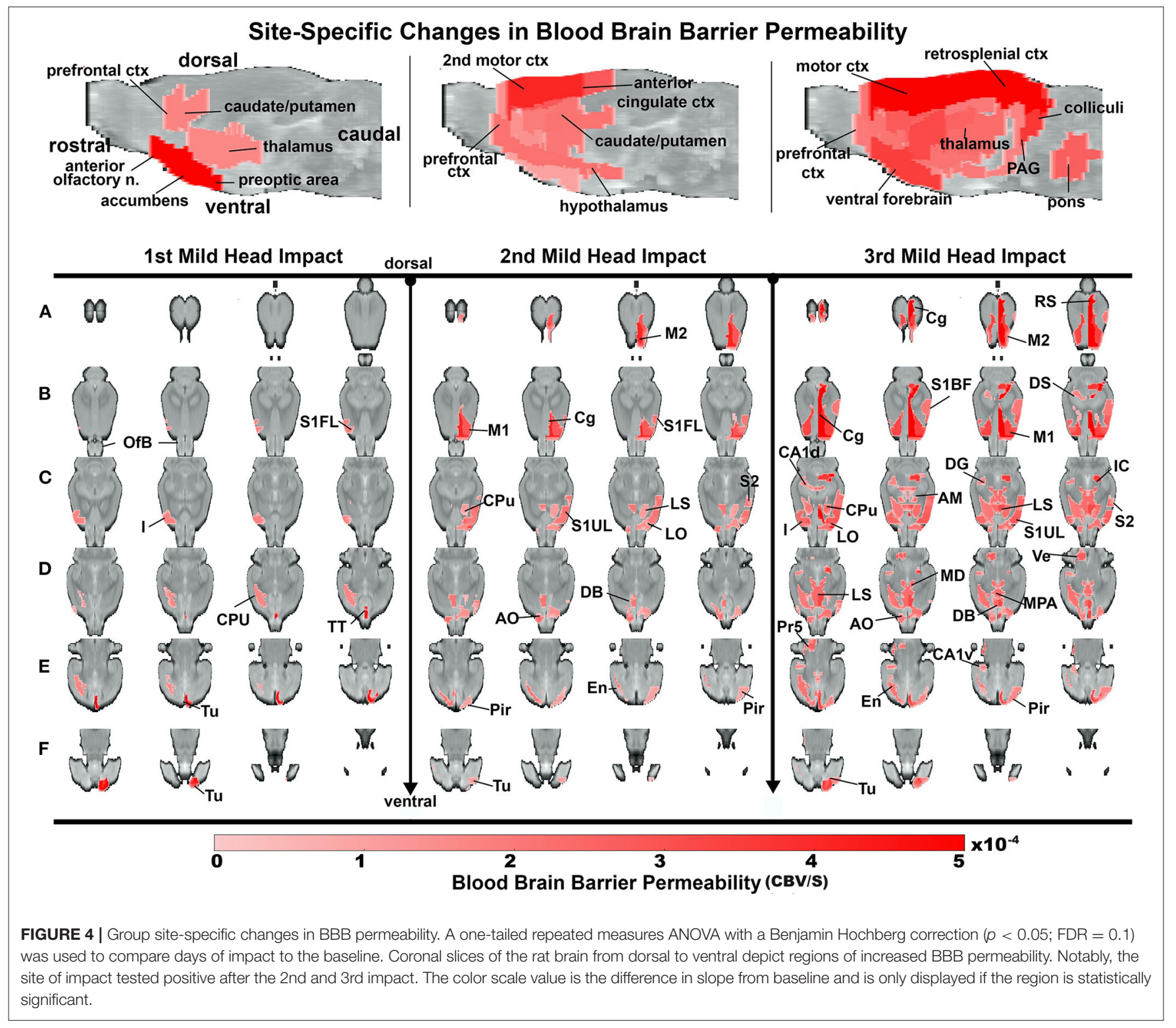

rate of mortality after multiple hits was ca $10 \%$. Minor deficits in balance and motor coordination recovered over time. There was no brain edema as determined by the wet/dry method to assess changes in water content. The cortex under the site of impact showed no staining for IgG suggesting there was no increase in $\mathrm{BBB}$ permeability. Reactive gliosis was observed in the cortex and hippocampus. Logsdon et al., reported two mild blast injuries, $12-15 \mathrm{~min}$ apart, caused an immediate increase in BBB permeability to radiolabeled albumin across much of the brain (21). The change in BBB permeability was biphasic, resolving within $24 \mathrm{~h}$ but reappearing $72 \mathrm{~h}$ later. The delayed response was region-specific, affecting the frontal cortex, hippocampus, thalamus, and medulla. The immediate and latent changes in $\mathrm{BBB}$ permeability could be explained by a reduction in the brain endothelial cell expression of tight junction protein, claudin5. Ren, and colleagues, developed a method ("hit and run") for giving a single mild head impact to mice without structural brain damage (20). There were no differences between sham and impacted mice for measures of cognitive behavior but there was a decrease in motor performance on the rotarod that persisted for 28 days. There was compelling evidence of diffuse gliosis with white matter degeneration that persists for 7-14 days. However, there was no edema or appreciable change in BBB permeability as measured by Evans Blue extravasation. Tagge et al. published a comprehensive study in over 300 mice on mild repetitive head injury using a closed head momentum exchange model in fully awake mice (22). Two head impacts separated by $15 \mathrm{~min}$, were followed by a battery of neurobehavioral tests, imaging, and histology for BBB permeability. There was no evidence of structural brain damage. After one impact, ca $82 \%$ of these mice ( $n=166 / 203$ ) exhibited minimal neurological impairment. Of these mice, $66.5 \%$ showed minimal or no impairment after the 
TABLE 1 | Site-specific changes in blood brain barrier permeability with repetitive mild head impacts.

\begin{tabular}{|c|c|c|c|c|c|c|}
\hline \multirow[b]{2}{*}{ Brain area } & \multicolumn{3}{|c|}{ Right brain } & \multicolumn{3}{|c|}{ Left brain } \\
\hline & 1st hit & 2nd hit & 3rd hit & 1st hit & 2nd hit & 3rd hit \\
\hline \multicolumn{7}{|c|}{ CHANGE IN BLOOD BRAIN BARRIER PERMEABILITY } \\
\hline Thalamus & & & 0.012 & & & 0.012 \\
\hline CA1 hippocampus & & & & & & 0.031 \\
\hline Anterior cingulate & & & 0.023 & & & \\
\hline $\begin{array}{l}\text { Medial striatum } \\
\text { caudate/putamen }\end{array}$ & & & 0.023 & & 0.031 & 0.040 \\
\hline $\begin{array}{l}\text { Anterior olfactory n., } \\
\text { endopiriform }\end{array}$ & & 0.002 & 0.025 & & & \\
\hline Septum & & 0.047 & 0.050 & & & 0.026 \\
\hline 2nd motor ctx & & & 0.007 & & 0.007 & 0.004 \\
\hline Primary motor ctx & & & & & 0.019 & 0.015 \\
\hline Prefrontal ctx & & & & & 0.011 & 0.010 \\
\hline Deep cerebellar n. & & & 0.007 & & & \\
\hline Rostral piriform ctx & & & & & 0.027 & 0.039 \\
\hline $\begin{array}{l}\text { Lateral striatum, } \\
\text { caudate/putamen }\end{array}$ & 0.038 & & 0.045 & & & \\
\hline $\begin{array}{l}\text { Subiculum } \\
\text { hippocampus }\end{array}$ & & & 0.023 & & & \\
\hline Inferior colliculus & & & & & & 0.033 \\
\hline Retrosplenial ctx & & & & & & 0.012 \\
\hline $\begin{array}{l}\text { Somatosensory ctx } \\
\text { barrel field }\end{array}$ & & & & & & 0.046 \\
\hline Somatosensory ctx jaw & 0.021 & & 0.029 & & 0.030 & 0.024 \\
\hline $\begin{array}{l}\text { Somatosensory ctx } \\
\text { upper lip }\end{array}$ & & & & & 0.022 & 0.018 \\
\hline $\begin{array}{l}\text { Olfactory tubercles, } \\
\text { tenia tecta }\end{array}$ & & & & 0.019 & & 0.037 \\
\hline
\end{tabular}

Shown are the $p$-values for the average change in BBB to the right or left side of the brain for specific brain areas following one, two, or three minor head impacts (hits).

2nd head impact. All animals recovered with $3 \mathrm{~h}$. At $24 \mathrm{~h}$ there was sparse gliosis. However, reactive astrocytosis was observed at 3 days and 2 weeks post injury characterized by ramified processes and swollen endfeet around small blood vessels at the cortical site of impact. Microgliosis appeared at 3 days but was mostly resolved by 2 weeks. This time course of neuropathology was also reported in mice hit only once. Fifty percentage of the mice showed no change in BBB permeability, while $40 \%$ showed minimal brain damage marked only by Evans blue extravasation. $\mathrm{BBB}$ permeability at the site of impact was noted within $3 \mathrm{~h}$ and persisted for 3 days. Pham et al. reported a single mild head impact in awake rats, again without structural brain damage, induced microgliosis at 1-, 3-, and 14-days post insult around impact and astrocytosis at day 3 in the hippocampus (57).

Measures of $\mathrm{BBB}$ permeability are variable across and within the different models described above. These studies on repetitive mild head impacts in mice, using different methods to inflict injury, all without structural brain damage, speak to the variability in behavioral and neurobiological outcomes. Judging these types of mild head impacts by changes in behavior would seem uninformative given the absence and/or transience of such measures across different models. The neuropathology, particularly gliosis would appear to offer a better biomarker as it is consistent in all models. The absence of any change in permeability with mild head impact may be due to the timing of the observation. Histological validation of changes in BBB permeability is a snapshot of a single time point of a dynamic process that is biphasic as described by Logsdon et al. (21), with an onset and duration noted by Tagge et al. (22). In a just published study using apparent diffusion coefficient from diffusion weighted imaging as a proxy of vasogenic edema from disruption in $\mathrm{BBB}$ permeability, we reported increased edema that peaked at $6 \mathrm{~h}$ post one mild head impact that resolved within $24 \mathrm{~h}$ (58). Hence post-mortem histology for assessing BBB permeability has its limitations set by the time from insult to animal sacrifice.

Dynamic contrast enhanced (DCE) MRI with gadoliniumbased contrast agents (GBCAs) is a method for assessing brain vascular permeability. However, the inaccuracy of DCE MRI is the major drawback. DCE looks at the rate of exchange of vascular contrast agent between plasma and the extracellular space of the brain parenchyma generating a volume transfer constant $\mathrm{K}_{\text {Trans }}$ (26). DCE MRI has been used to measure $\mathrm{BBB}$ permeability following moderate TBI in rodents (59-61). In humans, DCE MRI shows an increase in BBB permeability in response to moderate to severe TBI around damage tissue within $24 \mathrm{~h}$ of injury that persists for days (62). DCE MRI has also been used to evaluate vascular permeability in mild head injury. Athletes playing American football and thought to have mild, sub-concussive head injuries show evidence of increased BBB permeability (63). Using DCE-MRI, O'Keeffe and colleagues reported $\mathrm{BBB}$ disruption in response to repetitive mild concussions in rugby players after a season and mixed martial arts fighters within days after a competitive fight (64). In a just published study by Veksler et al., using a modified DCE MRI approach, they were able to generate maps of increased $\mathrm{BBB}$ permeability in American football players vs. controls (i.e., athletes from non-contact sports) that persisted for months after contact (65). The permeability maps were region specific, but primarily correlated with changes in white matter tracts. The permeability was characterized by a slow bloodto-brain transcellular transport mechanism underlying chronic BBB dysfunction. More recently, higher accuracy measurements have been achieved in rodents $(31,32)$ and humans $(33,34)$, however DCE-MRI remains limited to 2-dimensional imaging with thick slabs ( $1 \mathrm{~mm}$ in mice, $5 \mathrm{~mm}$ in humans) -in addition to requiring GBCAs.

QUTE-CE MRI was developed to generate quantitative vascular biomarkers for precision medicine (35-39). Ferumoxytol MRI with optimized 3D Ultra-Short Time-toecho (UTE) pulse sequences produces angiographic images unparalleled to time-of-flight imaging or GBCA first-pass imaging (see Figure 1, Supplementary Data). The contrast agent ferumoxytol, is an ultra-small superparamagnetic iron oxide nanoparticle (USPION) with a dextran coating. Since the size exceeds the cutoff $(\sim 6 \mathrm{~nm})$ for glomerular filtration, ferumoxytol is not cleared by the kidney, and instead is an 

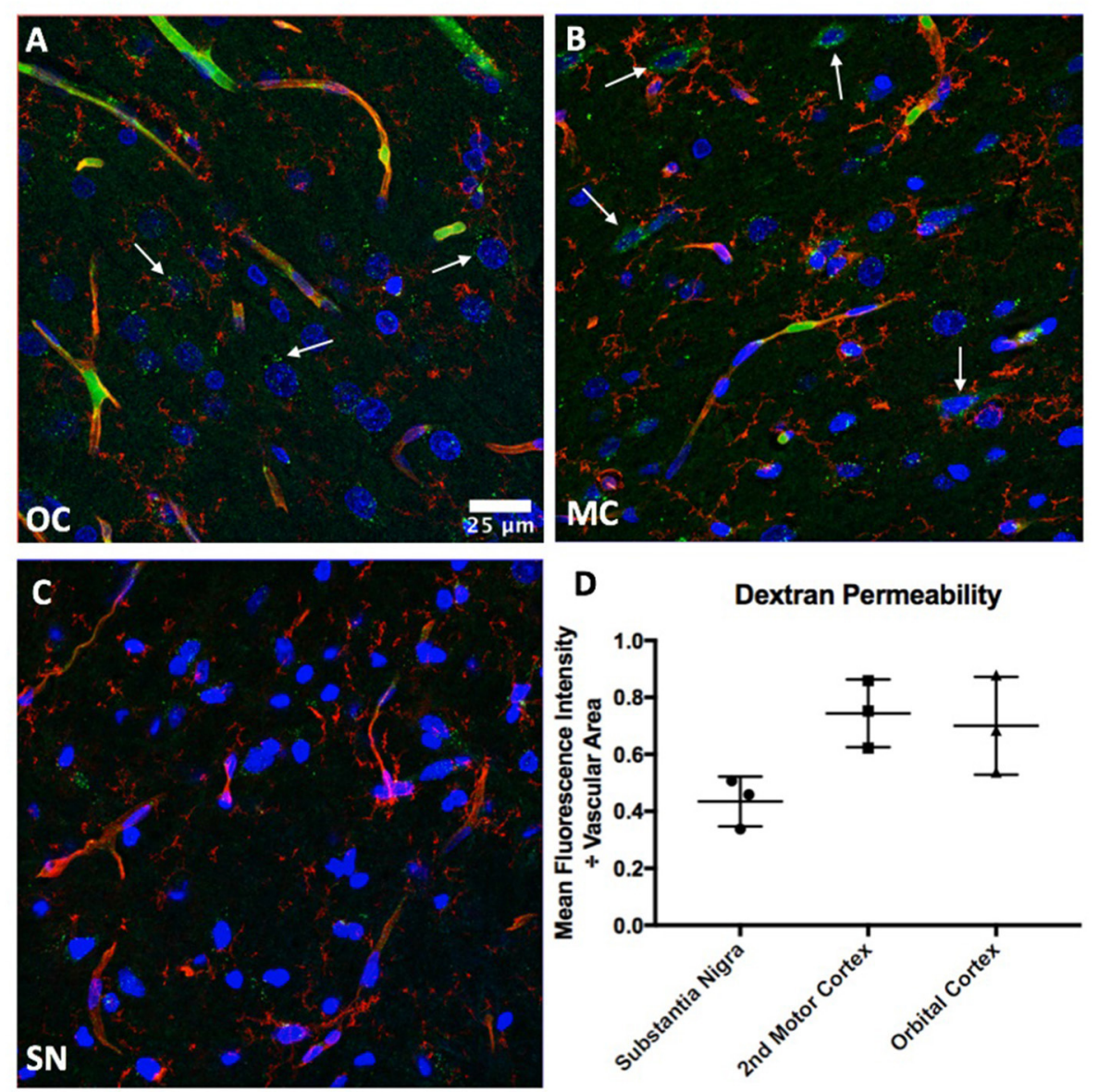

FIGURE 5 | Histological validation of BBB permeability. Fluorescent staining of the vasculature via Tomato lectin (red) demonstrates increases in FITC-dextran (green) accumulation within the parenchyma and vascular cells of the (A) orbital (OC) and (B) motor (MC) cortices, but not the (C) substantia nigra (SN). (D) Quantification of permeability. Nuclei are labeled in blue.

excellent blood pool contrast agent with a long intravascular half-life of $\sim 15 \mathrm{~h}$ (66). However, ferumoxytol has received an FDA black box warning for rare, but serious anaphylactic reactions. In clinical practice, the risk for anaphylaxis is now mitigated by administrating the agent over a 10-15-min infusion period-rather than a bolus (67). Nevertheless, development of an FDA approved hypoallergenic USPION formulation would be greatly beneficial for the safest clinical implementation of QUTE-CE MRI.

This model of repetitive head impact combined with noninvasive QUTE-CE enabled us to investigate two clinically relevant questions: (1) Does a single mild impact to the head compromise BBB integrity? (2) Do several mild impacts, within days of one another, exacerbate the risk of $\mathrm{BBB}$ injury? The question of whether the site-specific changes in $\mathrm{BBB}$ permeability would resolve over time or persist and evolve into cerebral vascular injury is unknown but could be followed by end-oflife studies using non-invasive imaging. In a recently published study using the three, mild head impact model described here, we reported alterations in indices of anisotropy reflecting white and gray matter damage and loss of functional connectivity between various brain regions 7-8 weeks post insult (43).

The imaging data confirmed that a single hit, did indeed, increase BBB permeability and that repeated impacts broadened the area of vulnerability to distal regions of the brain. The sensitivity of QUTE-CE to resolve insult in individual animals revealed the variability between subjects both in sensitivity to head impact and site-specific vulnerability. At baseline, all rats exhibited minimal leakage as measured with the method. While all rats were impacted on the dorsal cranium in the region of the forebrain, individual responses varied. Some rats were more resilient to head impact, as in the case of Rat 5 who responded primarily only after the 3rd impact, while others like Rats 1, 2, and 4 showed widespread increases in BBB permeability after a single head impact. All the head impacts included increased BBB permeability in the olfactory system and striatum, but injury to hindbrain areas was less predictable. Only after the 3rd impact was the hippocampus, CA1 and subiculum, affected. Each rat responded differently, an observation that in many ways reflects the human experience. 


\section{LIMITATIONS}

This was an exploratory study to determine whether QUTE$\mathrm{CE}$ imaging was sensitive enough to pick up changes in BBB permeability to mild head impacts, and as such, had several limitations. Foremost was a comparison to DCE the standard method used in the clinic to evaluate changes in $\mathrm{BBB}$ permeability, including examination of $\mathrm{BBB}$ leakage of nanoparticles versus macromolecular GBCAs. These studies were unfunded and exploratory and as such we did not have the resources to make a head-to-head comparison between QUTE$\mathrm{CE}$ and DCE. Also lacking in these studies was the use of females. We are aware of the many studies reporting sex differences in response to TBI (68-73). Recent reviews of the literature, report male/female differences in morbidity and mortality following TBI $(74,75)$. Also, the histology was only focused on a single fluorescent marker to show BBB permeability. Staining for markers of gliosis, neuron viability and capillary integrity would have provided a better understanding of the effect on mild head impact on the neurovascular unit. Lastly, it would have been interesting to know how long between impacts is necessary to avoid the compounded effect of multiple insults or the potential confound of CBV changes in tissue due to insult. This window of vulnerability was tested with intervals of $24 \mathrm{~h}$ only. Would 1 week or longer between impacts have negated the exacerbated increase in $\mathrm{BBB}$ permeability?

\section{SUMMARY}

Quantitative measures of increased BBB permeability with QUTE-CE MRI appears to be feasible, even in individual subjects, as assessed in this exploratory study with 5 animals. At baseline, minimal leakage was present, and multiple hits exacerbated BBB leakage, which appeared to peak after the second hit. QUTE-CE may thus be a method to non-invasively follow the consequences of mild head injury at the level of the microvasculature in individual subjects. Given the reports that BBB leakage may play a critical role in the pathophysiology of early $\mathrm{AD}$ (76) and cerebral

\section{REFERENCES}

1. Cassidy JD, Carroll LJ, Peloso PM, Borg J, von Holst H, Holm L, et al. Incidence, risk factors and prevention of mild traumatic brain injury: results of the WHO Collaborating Centre Task Force on Mild Traumatic Brain Injury. J Rehabil Med. (2004) (43 Suppl):28-60. doi: 10.1080/16501960410023732

2. Lovell MR, Collins MW, Iverson GL, Field M, Maroon JC, Cantu R, et al. Recovery from mild concussion in high school athletes. J Neurosurg. (2003) 98:296-301. doi: 10.3171/jns.2003.98.2.0296

3. Pellman EJ, Lovell MR, Viano DC, Casson IR. Concussion in professional football: recovery of NFL and high school athletes assessed by computerized neuropsychological testing-Part 12. Neurosurgery. (2006) 58:263-74; discussion 263-274. doi: 10.1227/01.NEU.0000200272.56192.62

4. Guskiewicz KM, McCrea M, Marshall SW, Cantu RC, Randolph C, Barr W, et al. Cumulative effects associated with recurrent concussion in collegiate football players: the NCAA Concussion study. JAMA. (2003) 290:254955. doi: 10.1001/jama.290.19.2549

5. Marar M, McIlvain NM, Fields SK, Comstock RD. Epidemiology of concussions among United States high school athletes in 20 sports. Am J Sports Med. (2012) 40:747-55. doi: 10.1177/0363546511435626 amyloid angiopathy (77) QUTE-CE may provide a sensitive method for early diagnosis and assement of treatment efficacy.

\section{DATA AVAILABILITY STATEMENT}

The original contributions presented in the study are included in the article/Supplementary Material, further inquiries can be directed to the corresponding author/s.

\section{ETHICS STATEMENT}

The animal study was reviewed and approved by IACUC Northeastern University.

\section{AUTHOR CONTRIBUTIONS}

CF, PK, EE, and CG concept, drafting, and interpretation. JQ, PK, $\mathrm{JL}$, and IH execution and analysis. All authors have contributed substantially to the manuscript and read and agreed to the published version of the manuscript.

\section{FUNDING}

This work was supported by an AHA pre-doctoral fellowship (18PRE33960461), NIH grant (K01-HL125499), HHMI funded Inclusive Excellence Award to Northeastern University (PI Ondrechen) and Imaginostics.

\section{ACKNOWLEDGMENTS}

Dr. Andrew Siedlecki for providing ferumoxytol.

\section{SUPPLEMENTARY MATERIAL}

The Supplementary Material for this article can be found online at: https://www.frontiersin.org/articles/10.3389/fneur. 2021.729464/full\#supplementary-material

6. De Beaumont L, Theoret H, Mongeon D, Messier J, Leclerc S, Tremblay $\mathrm{S}$, et al. Brain function decline in healthy retired athletes who sustained their last sports concussion in early adulthood. Brain. (2009) 132:695708. doi: 10.1093/brain/awn347

7. Gardner RC, Yaffe K. Epidemiology of mild traumatic brain injury and neurodegenerative disease. Mol Cell Neurosci. (2015) 66:75-80. doi: 10.1016/j.mcn.2015.03.001

8. Konrad C, Geburek AJ, Rist F, Blumenroth H, Fischer B, Husstedt I, et al. Long-term cognitive and emotional consequences of mild traumatic brain injury. Psychol Med. (2011) 41:1197-211. doi: 10.1017/S00332917100 01728

9. McKee AC, Cantu RC, Nowinski CJ, Hedley-Whyte ET, Gavett BE, Budson AE, et al. Chronic traumatic encephalopathy in athletes: progressive tauopathy after repetitive head injury. J Neuropathol Exp Neurol. (2009) 68:70935. doi: 10.1097/NEN.0b013e3181a9d503

10. Wardlaw JM. Blood-brain barrier and cerebral small vessel disease. J Neurol Sci. (2010) 299:66-71. doi: 10.1016/j.jns.2010.08.042

11. Cash A, Theus MH. Mechanisms of blood-brain barrier dysfunction in traumatic brain injury. Int J Mol Sci. (2020) 21:3344. doi: 10.3390/ijms21093344 
12. Held F, Morris AWJ, Pirici D, Niklass S, Sharp MMG, Garz C, et al. Vascular basement membrane alterations and beta-amyloid accumulations in an animal model of cerebral small vessel disease. Clin Sci. (2017) 131:100113. doi: 10.1042/CS20170004

13. Baldwin SA, Fugaccia I, Brown DR, Brown LV, Scheff SW. Blood-brain barrier breach following cortical contusion in the rat. J Neurosurg. (1996) 85:476-81. doi: 10.3171/jns.1996.85.3.0476

14. Habgood MD, Bye N, Dziegielewska KM, Ek CJ, Lane MA, Potter A, et al. Changes in blood-brain barrier permeability to large and small molecules following traumatic brain injury in mice. Eur J Neurosci. (2007) 25:2318. doi: 10.1111/j.1460-9568.2006.05275.x

15. Shapira Y, Setton D, Artru AA, Shohami E. Blood-brain barrier permeability, cerebral edema, and neurologic function after closed head injury in rats. Anesth Analg. (1993) 77:141-8. doi: 10.1213/00000539-19930700 $0-00028$

16. Shlosberg D, Benifla M, Kaufer D, Friedman A. Blood-brain barrier breakdown as a therapeutic target in traumatic brain injury. Nat Rev Neurol. (2010) 6:393-403. doi: 10.1038/nrneurol.2010.74

17. Shreiber DI, Smith DH, Meaney DF. Immediate in vivo response of the cortex and the blood-brain barrier following dynamic cortical deformation in the rat. Neurosci Lett. (1999) 259:5-8. doi: 10.1016/S0304-3940(98)0 0853-2

18. Hay JR, Johnson VE, Young AM, Smith DH, Stewart W. Blood-brain barrier disruption is an early event that may persist for many years after traumatic brain injury in humans. J Neuropathol Exp Neurol. (2015) 74:114757. doi: 10.1093/jnen/74.12.1147

19. Kane MJ, Angoa-Perez M, Briggs DI, Viano DC, Kreipke CW, Kuhn DM. A mouse model of human repetitive mild traumatic brain injury. J Neurosci Methods. (2012) 203:41-9. doi: 10.1016/j.jneumeth.2011.09.003

20. Ren Z, Iliff JJ, Yang L, Yang J, Chen X, Chen MJ, et al. 'Hit \& Run' model of closed-skull traumatic brain injury (TBI) reveals complex patterns of posttraumatic AQP4 dysregulation. J Cereb Blood Flow Metab. (2013) 33:83445. doi: $10.1038 /$ jcbfm.2013.30

21. Logsdon AF, Meabon JS, Cline MM, Bullock KM, Raskind MA, Peskind ER, et al. Blast exposure elicits blood-brain barrier disruption and repair mediated by tight junction integrity and nitric oxide dependent processes. Sci Rep. (2018) 8:11344. doi: 10.1038/s41598-018-29341-6

22. Tagge CA, Fisher AM, Minaeva OV, Gaudreau-Balderrama A, Moncaster JA, Zhang XL, et al. Concussion, microvascular injury, and early tauopathy in young athletes after impact head injury and an impact concussion mouse model. Brain. (2018) 141:422-58. doi: 10.1093/brain/awx350

23. Graves JC, Kreipke CW. Endothelin, cerebral blood flow, and traumatic brain injury: implications for a future therapeutic target. In: Kobeissy $\mathrm{FH}$, editor. Brain Neurotrauma: Molecular, Neuropsychological, Rehabilitation Aspects. Boca Raton, FL: CRC Press (2015).

24. Kim J, Whyte J, Patel S, Avants B, Europa E, Wang J, et al. Resting cerebral blood flow alterations in chronic traumatic brain injury: an arterial spin labeling perfusion FMRI study. J Neurotrauma. (2010) 27:1399411. doi: $10.1089 /$ neu.2009.1215

25. Wardlaw JM, Smith EE, Biessels GJ, Cordonnier C, Fazekas F, Frayne R, et al. Neuroimaging standards for research into small vessel disease and its contribution to ageing and neurodegeneration. Lancet Neurol. (2013) 12:822-38. doi: 10.1016/S1474-4422(13)70124-8

26. Heye AK, Culling RD, Valdes Hernandez Mdel C, Thrippleton MJ, Wardlaw JM. Assessment of blood-brain barrier disruption using dynamic contrastenhanced MRI. a systematic review. Neuroimage Clin. (2014) 6:26274. doi: 10.1016/j.nicl.2014.09.002

27. Thrippleton MJ, Backes WH, Sourbron S, Ingrisch M, van Osch MJP, Dichgans M, et al. Quantifying blood-brain barrier leakage in small vessel disease: review and consensus recommendations. Alzheimers Dement. (2019) 15:840-58. doi: 10.1016/j.jalz.2019.01.013

28. Schabel MC, Parker DL. Uncertainty and bias in contrast concentration measurements using spoiled gradient echo pulse sequences. Phys Med Biol. (2008) 53:2345-73. doi: 10.1088/0031-9155/53/9/010

29. Walker-Samuel S, Parker CC, Leach MO, Collins DJ. Reproducibility of reference tissue quantification of dynamic contrast-enhanced data: comparison with a fixed vascular input function. Phys Med Biol. (2007) 52:75-89. doi: 10.1088/0031-9155/52/1/006
30. Raja R, Rosenberg GA, Caprihan A. MRI measurements of blood-brain barrier function in dementia: a review of recent studies. Neuropharmacology. (2018) 134:259-71. doi: 10.1016/j.neuropharm.2017.10.034

31. Montagne A, Nikolakopoulou AM, Zhao Z, Sagare AP, Si G, Lazic D, et al. Pericyte degeneration causes white matter dysfunction in the mouse central nervous system. Nat Med. (2018) 24:326-37. doi: 10.1038/nm.4482

32. Nikolakopoulou AM, Montagne A, Kisler K, Dai Z, Wang Y, Huuskonen MT, et al. Pericyte loss leads to circulatory failure and pleiotrophin depletion causing neuron loss. Nat Neurosci. (2019) 22:1089-98. doi: 10.1038/s41593-019-0434-z

33. Montagne A, Nation DA, Sagare AP, Barisano G, Sweeney MD, Chakhoyan A, et al. APOE4 leads to blood-brain barrier dysfunction predicting cognitive decline. Nature. (2020) 581:71-6. doi: 10.1038/s41586-020-2247-3

34. Nation DA, Sweeney MD, Montagne A, Sagare AP, D'Orazio LM, Pachicano $M$, et al. Blood-brain barrier breakdown is an early biomarker of human cognitive dysfunction. Nat Med. (2019) 25:270-6. doi: 10.1038/s41591-018-0297-y

35. Gharagouzloo CA, McMahon PN, Sridhar S. Quantitative contrast-enhanced MRI with superparamagnetic nanoparticles using ultrashort time-to-echo pulse sequences. Magn Reson Med. (2015) 74:431-41. doi: 10.1002/mrm.25426

36. Gharagouzloo CA, Timms L, Qiao J, Fang Z, Nneji J, Pandya A, et al. Quantitative vascular neuroimaging of the rat brain using superparamagnetic nanoparticles: new insights on vascular organization and brain function. Neuroimage. (2017) 163:24-33. doi: 10.1016/j.neuroimage.2017.09.003

37. Gharagouzloo CA, Timms L, Qiao J, Fang Z, Nneji J, Pandya A, et al. Dataset on a 173 region awake resting state quantitative cerebral blood volume rat brain atlas and regional changes to cerebral blood volume under isoflurane anesthetization and CO. Data Brief. (2018) 17:3936. doi: 10.1016/j.dib.2018.01.021

38. Qiao J, Lawson CM, Rentrup KFG, Kulkarni P, Ferris CF. Evaluating bloodbrain barrier permeability in a rat model of type 2 diabetes. $J$ Transl Med. (2020) 18:256. doi: 10.1186/s12967-020-02428-3

39. van de Ven AL, Tangutoori S, Baldwin P, Qiao J, Gharagouzloo C, Seitzer N, et al. Nanoformulation of olaparib amplifies parp inhibition and sensitizes PTEN/TP53-deficient prostate cancer to radiation. Mol Cancer Ther. (2017) 16:1279-89. doi: 10.1158/1535-7163.MCT-16-0740

40. Kilkenny C, Browne W, Cuthill IC, Emerson M, Altman DG, NC3Rs Reporting Guidelines Working Group. Animal research: reporting in vivo experiments: the ARRIVE guidelines. Br J Pharmacol. (2010) 160:15779. doi: 10.1111/j.1476-5381.2010.00872.x

41. Viano DC, Hamberger A, Bolouri H, Saljo A. Concussion in professional football: animal model of brain injury-part 15. Neurosurgery. (2009) 64:116273; discussion 1173. doi: 10.1227/01.NEU.0000345863.99099.C7

42. Mychasiuk R, Hehar H, Candy S, Ma I, Esser MJ. The direction of the acceleration and rotational forces associated with mild traumatic brain injury in rodents effect behavioural and molecular outcomes. J Neurosci Methods. (2016) 257:168-78. doi: 10.1016/j.jneumeth.2015.10.002

43. Kulkarni P, Morrison TR, Cai X, Iriah S, Simon N, Sabrick J, et al. Neuroradiological changes following single or repetitive mild TBI. Front Syst Neurosci. (2019) 13:34. doi: 10.3389/fnsys.2019.00034

44. Natarajan R, Northrop N, Yamamoto B. Fluorescein Isothiocyanate (FITC)dextran extravasation as a measure of blood-brain barrier permeability. Curr Protoc Neurosci. (2017) 79:9 58 51-59 58 15. doi: 10.1002/cpns.25

45. Prins ML, Alexander D, Giza CC, Hovda DA. Repeated mild traumatic brain injury: mechanisms of cerebral vulnerability. J Neurotrauma. (2013) 30:30-8. doi: 10.1089/neu.2012.2399

46. Qin Y, Li GL, Xu XH, Sun ZY, Gu JW, Gao FB. Brain structure alterations and cognitive impairment following repetitive mild head impact: an in vivo MRI and behavioral study in rat. Behav Brain Res. (2018) 340:418. doi: 10.1016/j.bbr.2016.08.008

47. Weil ZM, Gaier KR, Karelina K. Injury timing alters metabolic, inflammatory and functional outcomes following repeated mild traumatic brain injury. Neurobiol Dis. (2014) 70:108-16. doi: 10.1016/j.nbd.2014.06.016

48. Yoshiyama Y, Uryu K, Higuchi M, Longhi L, Hoover R, Fujimoto $S$, et al. Enhanced neurofibrillary tangle formation, cerebral atrophy, and cognitive deficits induced by repetitive mild brain injury in a transgenic tauopathy mouse model. J Neurotrauma. (2005) 22:113441. doi: $10.1089 /$ neu.2005.22.1134 
49. Kenney K, Amyot F, Haber M, Pronger A, Bogoslovsky T, Moore C, et al. Cerebral vascular injury in traumatic brain injury. Exp Neurol. (2016) 275:353-66. doi: 10.1016/j.expneurol.2015.05.019

50. Zhou Y, Chen Q, Wang Y, Wu H, Xu W, Pan Y, et al. Persistent neurovascular unit dysfunction: pathophysiological substrate and trigger for late-onset neurodegeneration after traumatic brain injury. Front Neurosci. (2020) 14:581. doi: 10.3389/fnins.2020.00581

51. LoBue C, Munro C, Schaffert J, Didehbani N, Hart J, Batjer H, et al. Traumatic brain injury and risk of long-term brain changes, accumulation of pathological markers, and developing dementia: a review. J Alzheimers Dis. (2019) 70:62954. doi: 10.3233/JAD-190028

52. Zlokovic BV. Neurovascular pathways to neurodegeneration in Alzheimer's disease and other disorders. Nat Rev Neurosci. (2011) 12:723-38. doi: 10.1038/nrn3114

53. Jafari S, Etminan M, Aminzadeh F, Samii A. Head injury and risk of Parkinson disease: a systematic review and meta-analysis. Mov Disord. (2013) 28:12229. doi: $10.1002 / \mathrm{mds} .25458$

54. Franz CK, Joshi D, Daley EL, Grant RA, Dalamagkas K, Leung A, et al. Impact of traumatic brain injury on amyotrophic lateral sclerosis: from bedside to bench. J Neurophysiol. (2019) 122:1174-85. doi: 10.1152/jn.00572.2018

55. Management of Concussion/mTBI Working Group. Management of concussion/m, VA/DoD clinical practice guideline for management of concussion/mild traumatic brain injury. J Rehabil Res Dev. (2009) 46:1-68. doi: 10.1682/JRRD.2008.03.0038

56. Report to Congress on Traumatic Brain Injury in the United States: Epidemiology Rehabilitation. National Center for Injury Prevention and Control; Division of Unintentional Injury, Prevention, Centers for Disease, Control, and Prevention. Atlanta, GA (2018).

57. Pham L, Shultz SR, Kim HA, Brady RD, Wortman RC, Genders SG, et al. Mild closed-head injury in conscious rats causes transient neurobehavioral and glial disturbances: a novel experimental model of concussion. J Neurotrauma. (2019) 36:2260-71. doi: 10.1089/neu.2018.6169

58. Kulkarni P, Bhosle MR, Lu SF, Simon NS, Iriah S, Brownstein MJ, et al. Evidence of early vasogenic edema following minor head impact that can be reduced with a vasopressin V1a receptor antagonist. Brain Res Bull. (2020) 165:218-27. doi: 10.1016/j.brainresbull.2020.10.001

59. Li Y, Li M, Zuo L, Shi Q, Qin W, Yang L, et al. Compromised blood-brain barrier integrity is associated with total magnetic resonance imaging burden of cerebral small vessel disease. Front Neurol. (2018) 9:221. doi: 10.3389/fneur.2018.00221

60. Miller HA, Magsam AW, Tarudji AW, Romanova S, Weber L, Gee CC, et al. Evaluating differential nanoparticle accumulation and retention kinetics in a mouse model of traumatic brain injury via $\mathrm{K}$ (trans) mapping with MRI. Sci Rep. (2019) 9:16099. doi: 10.1038/s41598-019-52622-7

61. Yu M, Yang D, Wang M, Wei X, Li W. Early stage of diffusional kurtosis imaging and dynamic contrast-enhanced magnetic resonance imaging correlated with long-term neurocognitive function after experimental traumatic brain injury. Neurosci Lett. (2019) 705:206-11. doi: 10.1016/j.neulet.2019.04.034

62. Jungner M, Siemund R, Venturoli D, Reinstrup P, W SC, Bentzer P. Blood-brain barrier permeability following traumatic brain injury. Minerva Anestesiol. (2016) 82:525-33.

63. Weissberg I, Veksler R, Kamintsky L, Saar-Ashkenazy R, Milikovsky DZ, Shelef I, et al. Imaging blood-brain barrier dysfunction in football players. JAMA Neurol. (2014) 71:1453-5. doi: 10.1001/jamaneurol.2014.2682

64. O'Keeffe E, Kelly E, Liu Y, Giordano C, Wallace E, Hynes M, et al. Dynamic blood-brain barrier regulation in mild traumatic brain injury. J Neurotrauma. (2020) 37:347-56. doi: 10.1089/neu.2019.6483

65. Veksler R, Vazana U, Serlin Y, Prager O, Ofer J, Shemen N, et al. Slow blood-tobrain transport underlies enduring barrier dysfunction in American football players. Brain. (2020) 143:1826-42. doi: 10.1093/brain/awaa140

66. Bremerich J, Bilecen D, Reimer P. MR angiography with blood pool contrast agents. Eur Radiol. (2007) 17:3017-24. doi: 10.1007/s00330-007-0712-0
67. Varallyay CG, Toth GB, Fu R, Netto JP, Firkins J, Ambady P, et al. What does the boxed warning tell us? Safe practice of using ferumoxytol as an MRI contrast agent. AJNR Am J Neuroradiol. (2017) 38:1297302. doi: 10.3174/ajnr.A5188

68. Bazarian JJ, Blyth B, Mookerjee S, He H, McDermott MP. Sex differences in outcome after mild traumatic brain injury. J Neurotrauma. (2010) 27:52739. doi: 10.1089/neu.2009.1068

69. Broshek DK, Kaushik T, Freeman JR, Erlanger D, Webbe F, Barth JT. Sex differences in outcome following sports-related concussion. J Neurosurg. (2005) 102:856-63. doi: 10.3171/jns.2005.102.5.0856

70. Colantonio A, Harris JE, Ratcliff G, Chase S, Ellis K. Gender differences in self reported long term outcomes following moderate to severe traumatic brain injury. BMC Neurol. (2010) 10:102. doi: 10.1186/1471-237 7-10-102

71. Colvin AC, Mullen J, Lovell MR, West RV, Collins MW, Groh M. The role of concussion history and gender in recovery from soccer-related concussion. Am J Sports Med. (2009) 37:1699-1704. doi: 10.1177/03635465093 32497

72. Covassin T, Schatz P, Swanik CB. Sex differences in neuropsychological function and post-concussion symptoms of concussed collegiate athletes. Neurosurgery. (2007) 61:345-50; discussion 350341. doi: 10.1227/01.NEU.0000279972.95060.CB

73. Sandel NK, Schatz P, Goldberg KB, Lazar M. Sex-based differences in cognitive deficits and symptom reporting among acutely concussed adolescent lacrosse and soccer players. Am J Sports Med. (2017) 45:93744. doi: 10.1177/0363546516677246

74. Caplan HW, Cox CS, Bedi SS. Do microglia play a role in sex differences in TBI? J Neurosci Res. (2017) 95:509-17. doi: 10.1002/jnr.23854

75. Mollayeva T, Mollayeva S, Colantonio A. Traumatic brain injury: sex, gender and intersecting vulnerabilities. Nat Rev Neurol. (2018) 14:71122. doi: 10.1038/s41582-018-0091-y

76. van de Haar HJ, Burgmans S, Jansen JF, van Osch MJ, van Buchem MA, Muller $\mathrm{M}$, et al. Blood-brain barrier leakage in patients with early Alzheimer disease. Radiology. (2016) 281:527-35. doi: 10.1148/radiol.2016152244

77. Freeze WM, Bacskai BJ, Frosch MP, Jacobs HIL, Backes WH, Greenberg SM, et al. Blood-brain barrier leakage and microvascular lesions in cerebral amyloid angiopathy. Stroke. (2019) 50:32835. doi: 10.1161/STROKEAHA.118.023788

Conflict of Interest: CF has a financial interest in Animal Imaging Research, the company that makes the RF electronics and holders for animal imaging. CF, CG, and PK are inventors of QUTE-CE MRI related patents and CG is CEO of Imaginostics, a company created to translate the QUTE-CE MRI technology to the clinic.

The remaining authors declare that the research was conducted in the absence of any commercial or financial relationships that could be construed as a potential conflict of interest.

Publisher's Note: All claims expressed in this article are solely those of the authors and do not necessarily represent those of their affiliated organizations, or those of the publisher, the editors and the reviewers. Any product that may be evaluated in this article, or claim that may be made by its manufacturer, is not guaranteed or endorsed by the publisher.

Copyright (c) 2021 Leaston, Qiao, Harding, Kulkarni, Gharagouzloo, Ebong and Ferris. This is an open-access article distributed under the terms of the Creative Commons Attribution License (CC BY). The use, distribution or reproduction in other forums is permitted, provided the original author(s) and the copyright owner(s) are credited and that the original publication in this journal is cited, in accordance with accepted academic practice. No use, distribution or reproduction is permitted which does not comply with these terms. 